\title{
$P-v$ Criticality of a Specific Black Hole in $f(R)$ Gravity Coupled with Yang-Mills Field
}

\author{
Ali Övgün $\mathbb{B D}^{1,2,3}$ \\ ${ }^{1}$ Instituto de Física, Pontificia Universidad Católica de Valparaíso, Casilla Postal 4950, Valparaíso, Chile \\ ${ }^{2}$ Physics Department, Arts and Sciences Faculty, Eastern Mediterranean University, Famagusta, Northern Cyprus, Mersin 10, Turkey \\ ${ }^{3}$ TH Division, Physics Department, CERN, 1211 Geneva 23, Switzerland
}

Correspondence should be addressed to Ali Övgün; ali.ovgun@pucv.cl

Received 17 November 2017; Revised 18 January 2018; Accepted 30 January 2018; Published 25 June 2018

Academic Editor: Douglas Singleton

Copyright (c) 2018 Ali Övgün. This is an open access article distributed under the Creative Commons Attribution License, which permits unrestricted use, distribution, and reproduction in any medium, provided the original work is properly cited. The publication of this article was funded by SCOAP .

\begin{abstract}
We study the $P-v$ criticality of a specific charged AdS type black hole (SBH) in $f(R)$ gravity coupled with Yang-Mills field. In the extended phase space, we treat the cosmological constant as a thermodynamic pressure. After we study the various thermodynamical quantities, we show that the thermodynamic properties of the SBH behave as a Van der Waals liquid-gas system at the critical points and there is a first-order phase transition between small-large SBH.
\end{abstract}

\section{Introduction}

Important contribution on black holes' thermodynamics in anti-de-Sitter (AdS) spacetime is made by Hawking and Page [1], where a first-order phase transition is discovered between the Schwarzschild-anti-de-Sitter (SAdS) black holes that is known as the Hawking-Page transition. Then Chamblin et al. and Cvetic et al. show that the first-order phase transition among Reissner Nordstrom (RN) AdS black holes and the similarities between charged AdS black holes and liquid-gas systems in grand canonical ensemble [2-4]. Moreover, in the seminal papers of Kubiznak and Mann [5], the cosmological constant $\Lambda$ is used as dynamical pressure [6]

$$
P=-\frac{\Lambda}{8 \pi}=\frac{3}{8 \pi l^{2}}
$$

for the RN-AdS black holes in the extended phase space, instead of treating the $\Lambda$ as a fixed parameter (in standard thermodynamic), and its conjugate variable has dimension of volume

$$
V=\left(\frac{\partial M}{\partial P}\right)_{S, Q}
$$

Calculating the critical components and finding the phase transition of the RN-AdS black holes, it is shown that RN-AdS black holes behave similar to the Van der Waals fluid in the extended phase space where a first-order small/large black hole's phase transition occurs at a critical temperature below [7-11]. The Van der Waals equation,

$$
\left(P+\frac{a}{v^{2}}\right)(v-b)=k T,
$$

where its pressure is $P$, its temperature is $T$, its specific volume is $v=V / N$, the Boltzmann constant is $k$, and the positive constants are $a$ and $b$, takes into account the attractive and repulsive forces between molecules and gives an improved model for ideal gas behaviour to describe the basic properties of the liquid-gas phase transition with the ratio of $P_{c} V_{c} / T_{c}=3 / 8$ at critical points [12-17]. Afterwards, applications of the thermodynamical law's to the black hole's physics have gain attention. Different researches are done by using the variation of the first law of thermodynamics of black holes and also application of the $P-v$ criticality on black holes [18-88]. Furthermore, AdS-CFT correspondence is the other reason for studying the AdS black hole.

In this paper we use a black hole's solution in the YangMills field which is the one of the most interesting nonabelian gauge theories. By using the string theory models they find the Yang-Mills fields equations in low energy limit and then 
Yasskin found the first black hole solution in the theory of Yang-Mills coupled to Einstein theory [89].

Our main aim is to check $P-V$ criticality of a specific charged AdS type black hole $(\mathrm{SBH})$ in $f(R)$ gravity coupled with Yang-Mills field (YMF) [90] by comparing its result with the Van der Waals system. The Yang-Mills field is acted inside the nuclei with short range and $f(R)$ gravity which is an extension of Einstein's General Relativity with the arbitrary function of Ricci scalar $f(R)$ [91-94]. It would be of interest to study the $P-v$ criticality of SBH in $f(R)$ gravity coupled with YMF in the extended phase space treating the cosmological constant as a thermodynamic pressure. In this paper, we first study the thermodynamics in the extended phase space and then we obtain its critical exponents to show the existence of the Van der Waals like small-large black hole phase transitions.

The paper is organized as follows: in Section 2 we will briefly review the SBH in $f(R)$ gravity coupled with YMF. In Section $3 P-v$ criticality of the SBH in $f(R)$ gravity coupled with YMF will be studied in the extended phase space by calculating its critical exponents. In Section 4 we conclude with final remarks.

\section{SBH in $f(R)$ Gravity Coupled with YMF}

In this section, we briefly present a solution of SBH in $f(R)$ gravity coupled with YMF with a cosmological constant in $d$-dimensions [90]. Then we discuss its temperature, entropy, and other thermodynamic quantities. The action of the $f(R)$ gravity minimally coupled with $\mathrm{YMF}(c=G=1)$ is [90]

$$
\begin{gathered}
S=\int d^{d} x \sqrt{-g}\left[\frac{f(R)}{16 \pi}+\mathscr{L}(F)\right], \quad \text { (4) } \begin{array}{l}
\text { 0, it becomes well-known solutions in } f(R) \text { gravity. The } \\
\text { Bekenstein-Hawking temperature [95-98] of the black hole } \\
\text { is calculated by } T=\left.(1 / 4 \pi)(\partial f(r) / d r)\right|_{r=r_{h}}
\end{array} \\
T=\frac{(-1+(d-2) \ln (r)) Q^{d / 2-1 / 2}(d-1)(d-2)^{d / 2-1 / 2}(d-3)^{d / 4-1 / 4}+d 2^{d / 2-5 / 2}\left(-2 r^{2} \Lambda r^{d-2}+m(d-2)\right) \eta}{4 \pi r^{d-2} \eta d 2^{d / 2-5 / 2} r},
\end{gathered}
$$

where $f(R)$ is a function of the Ricci scalar $R$ and $\mathscr{L}(F)$ stands for the Lagrangian of the nonlinear YMF with $F=$ $(1 / 4) \operatorname{tr}\left(F_{\mu \nu}^{(a)} F^{(a) \mu \nu}\right)$, where the 2-form components of the YMF are $\mathbf{F}^{(a)}=(1 / 2) F_{\mu \nu}^{(a)} d x^{\mu} \wedge d x^{\nu}$. Here is the internal index $(a)$ for the degrees of freedom of the nonabelian YMF. It is noted that this nonlinear YMF can reduce to linear YM field $(\mathscr{L}(F)=$ $\left.-(1 / 4 \pi) F^{s}\right)$ for $s=1$ and $f_{R}=d f(R) / d R=\eta r$ which $\eta$ is an integration constant. Solving Einstein field equations for the $f(R)$ gravity coupled with YMF gives to the spherically symmetric black hole metrics (see equation (36) in [90])

$$
\begin{aligned}
d s^{2}= & -f(r) d t^{2}+\frac{d r^{2}}{f(r)} \\
& +r^{2}\left(d \theta_{1}^{2}+\sum_{i=2}^{d-2} \Pi_{j=1}^{i-1} \sin ^{2} \theta_{j} d \theta_{i}^{2}\right)
\end{aligned}
$$

with $0 \leq \theta_{d-2} \leq 2 \pi, 0 \leq \theta_{i} \leq \pi, 1 \leq i \leq d-3$, in which the metric function $f(r)$ is

$$
\begin{aligned}
& f(r) \\
& =\frac{d-3}{d-2}-\Lambda r^{2}-\frac{m}{r^{d-2}} \\
& \quad-\frac{(d-1)(d-2)^{(d-1) / 2}(d-3)^{(d-1) / 4}}{2^{(d-5) / 2} \eta d} \frac{Q^{(d-1) / 2} \ln r}{r^{d-2}} .
\end{aligned}
$$

Note that $\Lambda=-1 / l^{2}, M$ is the mass of the black hole, and $\eta$ is a constant. Furthermore for the limit of $Q^{7 / 2} \rightarrow$

where $r_{h}$ is the horizon of the black hole, and solving the equation $f\left(r_{h}\right)=0$, the total mass of the black hole is obtained as

$$
=-\frac{4 Q^{d / 2-1 / 2}(d / 2-1)^{d / 2} \ln (r) \sqrt{2}(d-1)(d-2)(d-3)^{d / 4-1 / 4}+\left(((f-1) d-2 f+3) r^{d-2}+r^{d} \Lambda(d-2)\right) d \sqrt{d-2} \eta}{\eta d(d-2)^{3 / 2}} .
$$

The entropy of the black hole can be derived as

$$
S=\frac{A_{h}}{4} \eta r_{h}
$$

where $A_{h}=((d-1) / \Gamma((d+1) / 2)) \pi^{(d-1) / 2} r_{h}^{d-2}$ is the area of the black hole's event horizon. Then, in the extended phase space, we calculate the pressure in terms of cosmological constant

$$
P=-\frac{\Lambda}{8 \pi}
$$

and its thermodynamic volume is

$$
V=\frac{\Omega_{d-2} r_{h}^{d-1} \eta}{n-1}
$$


where $\Omega_{d-2}$ is the volume of the unit sphere. Now the mass can be also written in terms of $P$ as follows:

$$
=-\frac{4 Q^{d / 2-1 / 2}(d / 2-1)^{d / 2} \ln (r) \sqrt{2}(d-1)(d-2)(d-3)^{d / 4-1 / 4}+\left(((f-1) d-2 f+3) r^{d-2}-8 r^{d} P \pi(d-2)\right) d \sqrt{d-2} \eta}{(d-2)^{3 / 2} \eta d} .
$$

The first law of the black hole thermodynamics in the extended phase space is

$$
d m=T d S+\Phi d Q+V d P,
$$

where the thermodynamic variables can be obtained as $T=$ $(\partial m / \partial S)_{\mathrm{Q}, P}, \Phi=(\partial m / \partial Q)_{S, P}$, and $V=(\partial m / \partial P)_{S, Q}$.

Then we write the generalized Smarr relation for the black hole, which can be derived also using the dimensional scaling, as

$$
m=2 T S+\Phi Q-2 V P .
$$

We introduce the cosmological constant as thermodynamic pressure in the extended phase space in (10), and it is seen that the first law of the black hole's thermodynamics and the Smarr relations is matched well.

\section{3. $P-v$ Criticality}

In this section, we investigate the critical behaviour of the $\mathrm{SBH}$ in the extended phase space. The critical point can be defined as

$$
\frac{\partial P}{\partial v}=\frac{\partial^{2} P}{\partial v^{2}}=0
$$

Now we consider the case of four dimensions $(d=4)$, where the metric function becomes

$$
f=\frac{1}{2}-\frac{m}{r^{2}}-r^{2} \Lambda-\frac{3 Q^{3 / 2} \ln (r)}{r^{2} \eta}
$$

and corresponding mass of the black hole is calculated as

$$
m=\frac{r^{2}}{2}-r^{2} \Lambda-\frac{3 Q^{3 / 2} \ln (r)}{\eta} .
$$

The temperature of the four dimensional $\mathrm{SBH}$ is

$$
T=-\frac{3 Q^{3 / 2}-r^{2} \eta+4 r^{4} \eta \Lambda}{4 \pi r^{3} \eta}
$$

Then we write the temperature in terms of $P(P=-\Lambda / 8 \pi)$ as follows:

$$
T=8 r P-\frac{3 Q^{3 / 2}}{4 r^{3} \eta \pi}+\frac{1}{4 r \pi}
$$

Afterwards one can easily obtain the pressure $P$ in terms of the temperature $T$ :

$$
P=\frac{T}{8 r}+\frac{3 Q^{3 / 2}}{32 r^{4} \eta \pi}-\frac{1}{32 r^{2} \pi} .
$$

To consider the $P-v$ criticality using the extended phase space, we write the black hole radius in terms of the specific volume $v$ as $r_{h}=(d-2) v / 4$. Using the condition of (15), we derive the critical Bekenstein-Hawking temperature $T_{c}$, critical pressure $P_{c}$, and critical specific volume $v_{c}$ as follows:

$$
\begin{aligned}
& T_{c}=-24 \frac{Q^{3 / 2}}{v^{3} \eta \pi}+\frac{1}{v \pi}, \\
& v_{c}=24 \frac{\sqrt{2} \sqrt{Q^{3 / 2}}}{\sqrt{\eta}}, \\
& P_{c}=\frac{\eta}{1152 Q^{3 / 2} \pi} .
\end{aligned}
$$

One can also find this relation which is same with a Van der Waals fluid

$$
\rho_{c}=\frac{P_{c} v_{c}}{T_{c}}=\frac{3}{8}
$$

It is noted that Figures 1 and 2 show that $P-r$ diagram is the same with the diagram of the Van der Waals liquid-gas system.

Let us now analyze the Gibbs free energy of the system. We first use the mass as enthalpy instead of internal energy and the Gibbs free energy in the extended phase space for the SBH in $f(R)$ gravity coupled with Yang-Mills field is calculated as

$$
\begin{aligned}
G & =m-T S \\
& =\frac{6 Q^{3 / 2}+r^{2} \eta-16 P \pi r^{4} \eta-18 Q^{3 / 2} \ln (r)}{6 \eta} .
\end{aligned}
$$

We plot the change of the free energy $G$ with $T$ in Figure 3. There is a small-large black hole phase transition as seen in Figure 3.

\section{Conclusion}

In this paper, we first treat the cosmological constant $\Lambda$ as a thermodynamical pressure $P$ and the thermodynamics 


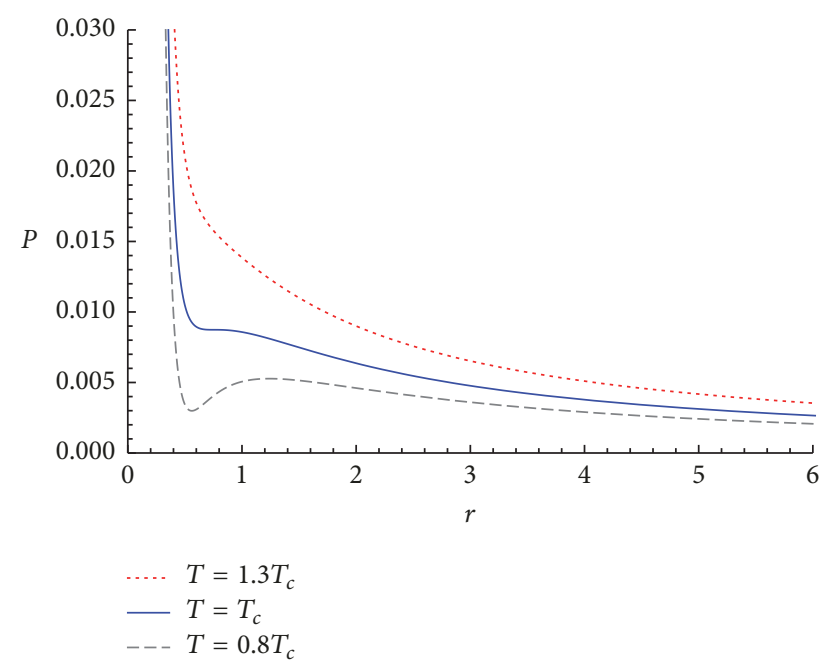

FIGURE 1: $P-r$ diagram of a SBH in a $f(R)$ gravity coupled with YMF for $Q=0.1$ and $\eta=1$.

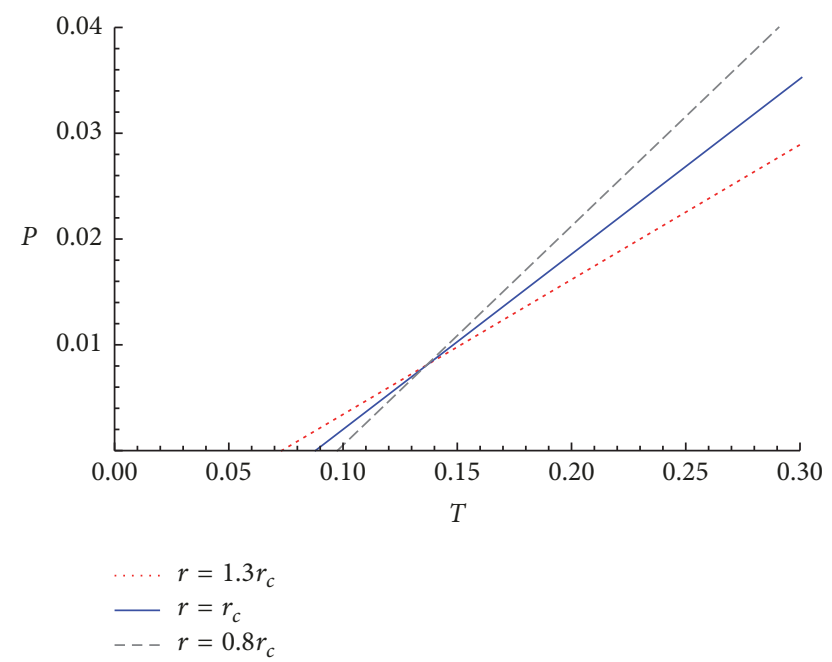

FIgURE 2: $P$-T diagram of a SBH in a $f(R)$ gravity coupled with YMF for $Q=0.1$ and $\eta=1$.

and $P-v$ criticality of the SBH in $f(R)$ gravity coupled with YMF is studied in the extended phase space. It is shown that there is a phase transition between small-large black holes. Furthermore, after we obtain the critical exponents, the critical behaviour of SBH in $f(R)$ gravity coupled with YMF in the extended space behaves also similarly as Van der Waals liquid-gas systems with the ratio of $P_{c} v_{c} / T_{c}=3 / 8$ at critical points. Hence it would be of great importance to obtain the $P-V$ criticality of SBH in $f(R)$ gravity coupled with YMF. Hence the critical ratio $P_{c} v_{c} / T_{c}=3 / 8$ is universal and independent from the modified gravities. The YMF has a parameter of $\eta$ but has no effect on the universal ratio of $3 / 8$.

It is also interesting to study the holographic duality of SBH in $f(R)$ gravity coupled with YMF. It is noted that without thermal fluctuations black hole is holographic dual with Van der Waals fluid given by $\left(P+a / V^{2}\right)(V-b)=T$, where $k$ is the Boltzmann constant $[84,85], b>0$ is nonzero

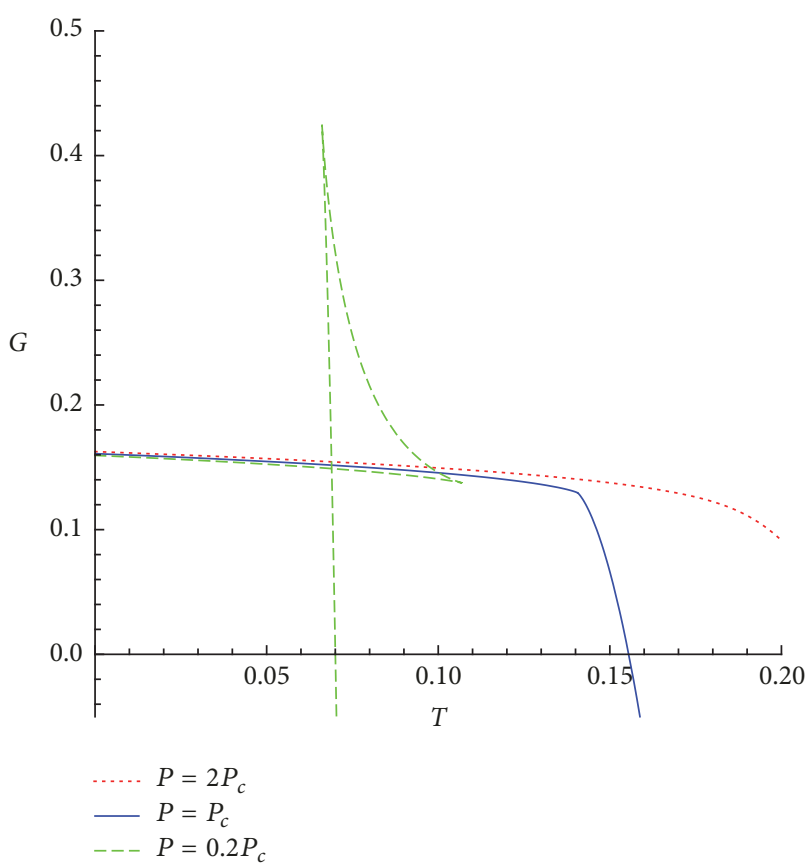

FIGURE 3: $G$-T diagram of a SBH in a $f(R)$ gravity coupled with YMF for different values of $P\left(P<P_{c}, P=P_{c}\right.$, and $\left.P>P_{c}\right)$ with $Q=0.1$ and $\eta=1$.

constant which is the size of the molecules of fluid, and the constant $a>0$ is a value of the interaction measurement between molecules. We leave this problem for the future projects.

\section{Conflicts of Interest}

The author declares that there are no conflicts of interest regarding the publication of this paper.

\section{Acknowledgments}

This work was supported by the Chilean FONDECYT Grant no. 3170035 (Ali Övgün). Ali Övgün is grateful to the CERN Theory (CERN-TH) Division, Waterloo University, Department of Physics and Astronomy, and also Perimeter Institute for Theoretical Physics for hosting him as a research visitor where part of this work was done. It is a pleasure to thank Professor Robert B. Mann for valuable discussions.

\section{References}

[1] S. W. Hawking and D. N. Page, "Thermodynamics of black holes in anti-de Sitter space," Communications in Mathematical Physics, vol. 87, no. 4, pp. 577-588, 1982/83.

[2] A. Chamblin, R. Emparan, C. V. Johnson, and R. C. Myers, "Charged AdS black holes and catastrophic holography," Physical Review D: Particles, Fields, Gravitation and Cosmology, vol. 60, no. 6, 1999.

[3] A. Chamblin, R. Emparan, C. V. Johnson, and R. C. Myers, "Holography, thermodynamics, and fluctuations of charged 
AdS black holes," Physical Review D: Particles, Fields, Gravitation and Cosmology, vol. 60, no. 10, Article ID 104026, 1999.

[4] M. Cvetic, G. W. Gibbons, D. Kubiznak, and C. N. Pope, "Black hole enthalpy and an entropy inequality for the thermodynamic volume," Physical Review D, vol. 84, 2011.

[5] D. Kubiznak and R. B. Mann, " $P-V$ criticality of charged AdS black holes," Journal of High Energy Physics, vol. 1207, article 33, 2012.

[6] B. P. Dolan, "The cosmological constant and black-hole thermodynamic potentials," Classical and Quantum Gravity, vol. 28, no. 12, Article ID 125020, 2011.

[7] S. Gunasekaran, D. Kubiznak, and R. B. Mann, "Extended phase space thermodynamics for charged and rotating black holes and Born-Infeld vacuum polarization," Journal of High Energy Physics, vol. 2012, no. 11, article 110, 2012.

[8] D. Kubiznak and R. B. Mann, "Black hole chemistry," Can J. Phys, vol. 93, no. 9, p. 999, 2015.

[9] D. Kubiznak, R. B. Mann, and M. Teo, "Black hole chemistry: thermodynamics with lambda," Classical and Quantum Gravity, vol. 34, no. 6, 2017.

[10] R. A. Hennigar, R. B. Mann, and E. Tjoa, "Superfluid black holes," Physical Review Letters, vol. 118, no. 2, Article ID 021301, 2017.

[11] A. M. Frassino, R. B. Mann, and F. Simovic, https://arxiv.org/ abs/1611.03525.

[12] R. A. Hennigar, E. Tjoa, and R. B. Mann, "Thermodynamics of hairy black holes in Lovelock gravity," Journal of High Energy Physics, vol. 2017, no. 2, article no. 70, 2017.

[13] S. H. Hendi, R. B. Mann, S. Panahiyan, and B. Eslam Panah, "Van der Waals like behavior of topological AdS black holes in massive gravity," Physical Review D: Particles, Fields, Gravitation and Cosmology, vol. 95, no. 2, Article ID 021501, 2017.

[14] D. Hansen, D. Kubiznak, and R. B. Mann, "Universality of $P$ $V$ Criticality in Horizon Thermodynamics," Journal of High Energy Physics, vol. 1701, no. 47, 2017.

[15] R. B. Mann, The Chemistry of Black Holes, vol. 170, Springer Proc.Phys., 2016.

[16] R. A. Hennigar, W. G. Brenna, and R. B. Mann, "P-v criticality in quasitopological gravity," Journal of High Energy Physics, vol. 2015, no. 7, article 77, pp. 1-31, 2015.

[17] T. Delsate and R. Mann, "Van Der Waals black holes in d dimensions," Journal of High Energy Physics, vol. 2015, no. 2, article no. 70, 2015.

[18] S. Chen, X. Liu, C. Liu, and J. Jing, " $P-V$ criticality of AdS black hole in $f(R)$ gravity," Chinese Physics Letters, vol. 30, no. 6, Article ID 060401, 2013.

[19] R.-G. Cai, L.-M. Cao, L. Li, and R.-Q. Yang, "P-V criticality in the extended phase space of Gauss-Bonnet black holes in AdS space," Journal of High Energy Physics, vol. 2013, no. 9, article 5, 2013.

[20] A. Belhaj, M. Chabab, H. E. Moumni, and M. B. Sedra, "On thermodynamics of AdS black holes in arbitrary dimensions," Chinese Physics Letters, vol. 29, no. 10, Article ID 100401, 2012.

[21] K. Bhattacharya, B. R. Majhi, and S. Samanta, "van der Waals criticality in AdS black holes: A phenomenological study," Physical Review D: Particles, Fields, Gravitation and Cosmology, vol. 96, no. 8, 2017.

[22] K. Jafarzade and J. Sadeghi, "The thermodynamic efficiency in static and dynamic black holes," International Journal of Theoretical Physics, vol. 56, no. 11, pp. 3387-3399, 2017.
[23] J. Sadeghi and A. S. Kubeka, "P-V criticality of modified BTZ black hole," International Journal of Theoretical Physics, vol. 55, no. 5, pp. 2455-2459, 2016.

[24] J. Liang, "The P-v Criticality of a Noncommutative GeometryInspired Schwarzschild-AdS Black Hole," Chinese Physics Letters, vol. 34, no. 8, Article ID 080402, 2017.

[25] P. Pradhan, "Enthalpy, geometric volume and logarithmic correction to entropy for van der Waals black hole," EPL (Europhysics Letters), vol. 116, no. 1, Article ID 10001, 2016.

[26] X.-X. Zeng and L.-F. Li, "Holographic phase transition probed by nonlocal observables," Advances in High Energy Physics, vol. 2016, Article ID 6153435, 2016.

[27] J.-X. Mo, G.-Q. Li, and X.-B. Xu, "Combined effects of $\mathrm{f}(\mathrm{R})$ gravity and conformally invariant Maxwell field on the extended phase space thermodynamics of higher-dimensional black holes," The European Physical Journal C, vol. 76, no. 10, article no. 545, 2016.

[28] B. R. Majhi and S. Samanta, "P-V criticality of AdS black holes in a general framework," Physics Letters. B. Particle Physics, Nuclear Physics and Cosmology, vol. 773, pp. 203-207, 2017.

[29] Z.-Y. Fan, "Critical phenomena of regular black holes in anti-de Sitter space-time," The European Physical Journal C, vol. 77, no. 4, article no. 266, 2017.

[30] Y. G. Miao and Y. M. Wu, "Thermodynamics of the Schwarzschild-AdS black hole with a minimal length," Advances in High Energy Physics, vol. 2017, 14 pages, 2017.

[31] S. He, L. F. Li, and X. X. Zeng, "Holographic van der waals-like phase transition in the Gauss-Bonnet gravity," Nuclear Physics $B$, vol. 915, pp. 243-261, 2017.

[32] A. Mandal, S. Samanta, and B. R. Majhi, "Phase transition and critical phenomena of black holes: A general approach," Physical Review D: Particles, Fields, Gravitation and Cosmology, vol. 94, no. 6, Article ID 064069, 2016.

[33] D. Chen, G. qingyu, and J. Tao, "The modified first laws of thermodynamics of anti-de Sitter and de Sitter space-times," Nuclear Physics B, vol. 918, pp. 115-128, 2017.

[34] A. Dehyadegari, A. Sheykhi, and A. Montakhab, "Critical behavior and microscopic structure of charged AdS black holes via an alternative phase space," Physics Letters $B$, vol. 768, pp. 235-240, 2017.

[35] S. H. Hendi, S. Panahiyan, B. Eslam Panah, M. Faizal, and M. Momennia, "Critical behavior of charged black holes in GaussBonnet gravity's rainbow," Physical Review D: Particles, Fields, Gravitation and Cosmology, vol. 94, no. 2, Article ID 024028, 2016.

[36] Y.-B. Ma, R. Zhao, and S. Cao, "Q- $\Phi$ criticality in the extended phase space of $(\mathrm{n}+1)$-dimensional RN-AdS black holes," The European Physical Journal C, vol. 76, no. 12, article no. 669, 2016.

[37] H. Liu and X.-H. Meng, "P-V criticality in the extended phasespace of charged accelerating AdS black holes," Modern Physics Letters A, vol. 31, no. 37, Article ID 1650199, 2016.

[38] M. B. J. Poshteh and N. Riazi, "Phase transition and thermodynamic stability in extended phase space and charged Hořava-Lifshitz black holes," General Relativity and Gravitation, vol. 49, no. 5, article no. 64, 2017.

[39] X. Guo, H. Li, L. Zhang, and R. Zhao, "The critical phenomena of charged rotating de Sitter black holes," Classical and Quantum Gravity, vol. 33, no. 13, Article ID 135004, 2016.

[40] H.-F. Li, M.-S. Ma, and Y.-Q. Ma, "Thermodynamic properties of black holes in de Sitter space," Modern Physics Letters A, vol. 32, no. 2, 1750017, 12 pages, 2017. 
[41] M.-S. Ma and R.-H. Wang, "Peculiar P-V criticality of topological Hořava-Lifshitz black holes," Physical Review D: Particles, Fields, Gravitation and Cosmology, vol. 96, no. 2, Article ID 024052, 2017.

[42] S. Upadhyay, B. Pourhassan, and H. Farahani, "P-V criticality of first-order entropy corrected AdS black holes in massive gravity," Physical Review D: Particles, Fields, Gravitation and Cosmology, vol. 95, no. 10, Article ID 106014, 2017.

[43] S. Fernando, "Phase transitions of black holes in massive gravity," Modern Physics Letters A, vol. 31, no. 16, Article ID 1650096, 2016.

[44] S. Fernando, "P-V criticality in AdS black holes of massive gravity," Physical Review D: Particles, Fields, Gravitation and Cosmology, vol. 94, no. 12, Article ID 124049, 2016.

[45] J. Sadeghi, B. Pourhassan, and M. Rostami, "P-V criticality of logarithm-corrected dyonic charged AdS black holes," Physical Review D: Particles, Fields, Gravitation and Cosmology, vol. 94, no. 6, Article ID 064006, 2016.

[46] Y.-G. Miao and Z.-M. Xu, "Phase transition and entropy inequality of noncommutative black holes in a new extended phase space," Journal of Cosmology and Astroparticle Physics, vol. 2017, no. 3, article no. 046, 2017.

[47] P. Cheng, S.-W. Wei, and Y.-X. Liu, "Critical phenomena in the extended phase space of Kerr-Newman-AdS black holes," Physical Review D: Particles, Fields, Gravitation and Cosmology, vol. 94, no. 2, Article ID 024025, 2016.

[48] X. Guo, H. Li, L. Zhang, and R. Zhao, "The phase transition of higher dimensional charged black holes," Advances in High Energy Physics, vol. 2016, Article ID 7831054, 10 pages, 2016.

[49] J. Liang, C.-B. Sun, and H.-T. Feng, "P-V criticality in the extended phase space of charged $\mathrm{f}(\mathrm{R})$ black holes in AdS spacetime," EPL (Europhysics Letters), vol. 113, no. 3, Article ID 30008, 2016.

[50] L. C. Zhang and R. Zhao, "The critical phenomena of Schwarzschild-de Sitter black hole," EPL (Europhysics Letters), vol. 113, no. 1, Article ID 10008, 2016.

[51] J.-X. Mo, G.-Q. Li, and X.-B. Xu, "Effects of power-law Maxwell field on the critical phenomena of higher dimensional dilaton black holes," Physical Review D: Particles, Fields, Gravitation and Cosmology, vol. 93, no. 8, Article ID 084041, 2016.

[52] M. H. Dehghani, A. Sheykhi, and Z. Dayyani, "Critical behavior of Born-Infeld dilaton black holes," Physical Review D: Particles, Fields, Gravitation and Cosmology, vol. 93, no. 2, Article ID 024022, 2016.

[53] X. X. Zeng and L. F. Li, "Van der waals phase transition in the framework of holography," Physics Letters B, vol. 764, pp. 100108, 2017.

[54] R. Maity, P. Roy, and T. Sarkar, "Black hole phase transitions and the chemical potential," Physics Letters B, vol. 765, pp. 386-394, 2017.

[55] S. H. Hendi, R. M. Tad, Z. Armanfard, and M. S. Talezadeh, "Extended phase space thermodynamics and P-V criticality: brans-dicke-born-infeld vs. einstein-born-infeld-dilaton black holes," The European Physical Journal C, vol. 76, no. 5, article 263, 2016.

[56] A. Karch and B. Robinson, "Holographic black hole chemistry," Journal of High Energy Physics, vol. 2015, no. 12, article no. 73, pp. 1-15, 2015.

[57] S.-W. Wei, P. Cheng, and Y.-X. Liu, "Analytical and exact critical phenomena of d -dimensional singly spinning Kerr-AdS black holes," Physical Review D: Particles, Fields, Gravitation and Cosmology, vol. 93, no. 8, Article ID 084015, 2016.
[58] J. Xu, L.-M. Cao, and Y.-P. Hu, "P-V criticality in the extended phase space of black holes in massive gravity," Physical Review D: Particles, Fields, Gravitation and Cosmology, vol. 91, no. 12, Article ID 124033, 2015.

[59] J.-X. Mo and W.-B. Liu, "hase transitions, geometrothermodynamics, and critical exponents of black holes with conformal anomaly," Advances in High Energy Physics, vol. 2014, Article ID 739454, 10 pages, 2014.

[60] S. H. Hendi and Z. Armanfard, "Extended phase space thermodynamics and $\mathrm{P}-\mathrm{V}$ criticality of charged black holes in Brans-Dicke theory," General Relativity and Gravitation, vol. 47, no. 10, article no. 125, 2015.

[61] Z. Sherkatghanad, B. Mirza, Z. Mirzaiyan, and S. A. H. Mansoori, "Critical behaviors and phase transitions of black holes in higher order gravities and extended phase spaces," International Journal of Modern Physics D, vol. 26, no. 3, Article ID 1750017, 2017.

[62] M. Zhang, Z.-Y. Yang, D.-C. Zou, W. Xu, and R.-H. Yue, "P-V criticality of AdS black hole in the Einstein-Maxwell-powerYang-Mills gravity," General Relativity and Gravitation, vol. 47, no. 2, 2015.

[63] M. H. Dehghani, S. Kamrani, and A. Sheykhi, "P-V criticality of charged dilatonic black holes," Physical Review D: Particles, Fields, Gravitation and Cosmology, vol. 90, no. 10, Article ID 104020, 2014.

[64] S. H. Hendi, S. Panahiyan, and B. Eslam Panah, "P - V criticality and geometrical thermodynamics of black holes with BornInfeld type nonlinear electrodynamics," International Journal of Modern Physics D, vol. 25, no. 1, Article ID 1650010, 2016.

[65] D.-C. Zou, S.-J. Zhang, and B. Wang, "Critical behavior of BornInfeld AdS black holes in the extended phase space thermodynamics," Physical Review D: Particles, Fields, Gravitation and Cosmology, vol. 89, Article ID 044002, 2014.

[66] D. C. Zou, Y. Liu, and B. Wang, "Critical behavior of charged Gauss-Bonnet-AdS black holes in the grand canonical ensemble," Physical Review D: Particles, Fields, Gravitation and Cosmology, vol. 90, no. 4, Article ID 044063, 2014.

[67] M. Zhang, D.-C. Zou, and R.-H. Yue, "Reentrant phase transitions and triple points of topological AdS black holes in BornInfeld-massive gravity," Advances in High Energy Physics, Art. ID 3819246, 11 pages, 2017.

[68] D. Zou, Y. Liu, and R. Yue, "Behavior of quasinormal modes and Van der Waals-like phase transition of charged AdS black holes in massive gravity," The European Physical Journal C, vol. 77, no. 6, 2017.

[69] D. Zou, R. Yue, and M. Zhang, "Reentrant phase transitions of higher-dimensional AdS black holes in dRGT massive gravity," The European Physical Journal C, vol. 77, no. 4, 2017.

[70] C.-Y. Zhang, S.-J. Zhang, D.-C. Zou, and B. Wang, "Charged scalar gravitational collapse in de Sitter spacetime," Physical Review D: Particles, Fields, Gravitation and Cosmology, vol. 93, no. 6, Article ID 064036, 2016.

[71] D.-C. Zou, Y. Liu, C.-Y. Zhang, and B. Wang, "Dynamical probe of thermodynamical properties in three-dimensional hairy AdS black holes," EPL (Europhysics Letters), vol. 116, no. 4, Article ID 40005, 2016.

[72] D.-C. Zou, Y. Liu, B. Wang, and W. Xu, "Thermodynamics of rotating black holes with scalar hair in three dimensions," Physical Review D: Particles, Fields, Gravitation and Cosmology, vol. 90, no. 10, Article ID 104035, 2014.

[73] H.-H. Zhao, L.-C. Zhang, M.-S. Ma, and R. Zhao, " $P$ - V criticality of higher dimensional charged topological dilaton de Sitter 
black holes," Physical Review D: Particles, Fields, Gravitation and Cosmology, vol. 90, no. 6, Article ID 064018, 2014.

[74] G.-Q. Li, "Effects of dark energy on $P-V$ criticality of charged AdS black holes," Physics Letters B, vol. 735, pp. 256-260, 2014.

[75] J.-X. Mo and W.-B. Liu, " $P-V$ criticality of topological black holes in Lovelock-Born-Infeld gravity," The European Physical Journal C, vol. 74, article 2836, 2014.

[76] R. Zhao, H. H. Zhao, M. S. Ma, and L. C. Zhang, "On the critical phenomena and thermodynamics of charged topological dilaton AdS black holes," The European Physical Journal C, vol. 73, no. 12, pp. 1-10, 2013.

[77] X. Kuang and O. Miskovic, "Thermal phase transitions of dimensionally continued AdS black holes," Physical Review D: Particles, Fields, Gravitation and Cosmology, vol. 95, no. 4, 2017.

[78] X.-M. Kuang and J.-P. Wu, "Effect of quintessence on holographic fermionic spectrum," The European Physical Journal C, vol. 77, no. 10, article no. 670, 2017.

[79] C.-J. Luo, X.-M. Kuang, and F.-W. Shu, "Charged Lifshitz black hole and probed Lorentz-violation fermions from holography," Physics Letters B, vol. 769, pp. 7-13, 2017.

[80] L. Aránguiz, X.-M. Kuang, and O. Miskovic, "Topological black holes in pure Gauss-Bonnet gravity and phase transitions," Physical Review D: Particles, Fields, Gravitation and Cosmology, vol. 93, no. 6, Article ID 064039, 2016.

[81] D. Kastor, S. Ray, and J. Traschen, "Enthalpy and the mechanics of AdS black holes," Classical and Quantum Gravity, vol. 26, no. 19, Article ID 195011, 2009.

[82] M. Azreg-Aïnou, "Black hole thermodynamics: no inconsistency via the inclusion of the missing P-V terms," Physical Review D: Particles, Fields, Gravitation and Cosmology, vol. 91, no. 6, Article ID 064049, 2015.

[83] M. Azreg-Aïnou, "Charged de Sitter-like black holes: quintessence-dependent enthalpy and new extreme solutions," The European Physical Journal C, vol. 75, no. 1, pp. 1-13, 2015.

[84] E. Caceres, P. H. Nguyen, and J. F. Pedraza, "Holographic entanglement entropy and the extended phase structure of STU black holes," Journal of High Energy Physics, vol. 2015, no. 9, article no. 184, 2015.

[85] C. V. Johnson, "Holographic heat engines," Classical and Quantum Gravity, vol. 31, no. 20, p. 205002, 2014.

[86] M. Momennia, "Reentrant phase transition of Born-Infelddilaton black holes," https://arxiv.org/abs/1709.09039.

[87] S. H. Hendi and M. Momennia, "AdS charged black holes in Einstein-Yang-Mills gravity's rainbow: Thermal stability and P - V criticality," Physics Letters B, vol. 777, pp. 222-234, 2018.

[88] S. H. Hendi, B. Eslam Panah, S. Panahiyan, and M. Momennia, "Three dimensional magnetic solutions in massive gravity with (non)linear field," Physics Letters. B. Particle Physics, Nuclear Physics and Cosmology, vol. 775, pp. 251-261, 2017.

[89] P. B. Yasskin, "Solutions for gravity coupled to massless gauge fields," Physical Review D: Particles, Fields, Gravitation and Cosmology, vol. 12, no. 8, pp. 2212-2217, 1975.

[90] S. H. Mazharimousavi and M. Halilsoy, "Black hole solutions in ," Physical Review D: Particles, Fields, Gravitation and Cosmology, vol. 84, no. 6, 2011.

[91] S. Chakraborty and S. SenGupta, "Spherically symmetric brane spacetime with bulk $\mathrm{f}(\mathrm{R})$ gravity," The European Physical Journal C, vol. 75, no. 1, article no. 11, 2015.

[92] S. Nojiri and S. D. Odintsov, "Anti-evaporation of Schwarzschild-de Sitter black holes in F(R) gravity," Classical and Quantum Gravity, vol. 30, no. 12, Article ID 125003, 2013.
[93] S. Nojiri and S. D. Odintsov, "Instabilities and anti-evaporation of Reissner-Nordström black holes in modified $F(R)$ gravity," Physics Letters B, vol. 735, pp. 376-382, 2014.

[94] S. Nojiri and S. Odintsov, "Regular multihorizon black holes in modified gravity with nonlinear electrodynamics," Physical Review D: Particles, Fields, Gravitation and Cosmology, vol. 96, no. 10, 2017.

[95] I. Sakalli, A. Övgün, and K. Jusufi, "GUP assisted Hawking radiation of rotating acoustic black holes," Astrophysics and Space Science, vol. 361, no. 10, article no. 330, 2016.

[96] I. Sakalli and A. Övgün, "Black hole radiation of massive spin2 particles in $(3+1)$ dimensions," The European Physical Journal Plus, vol. 131, no. 6, article no. 184, 2016.

[97] I. Sakalli and A. Övgün, "Quantum tunneling of massive spin1 particles from non-stationary metrics," General Relativity and Gravitation, vol. 48, no. 1, article no. 1, pp. 1-10, 2016.

[98] I. Sakalli and A. Ovgun, "Tunnelling of vector particles from Lorentzian wormholes in 3+1 dimensions," The European Physical Journal Plus, vol. 130, no. 6, article 110, 2015. 

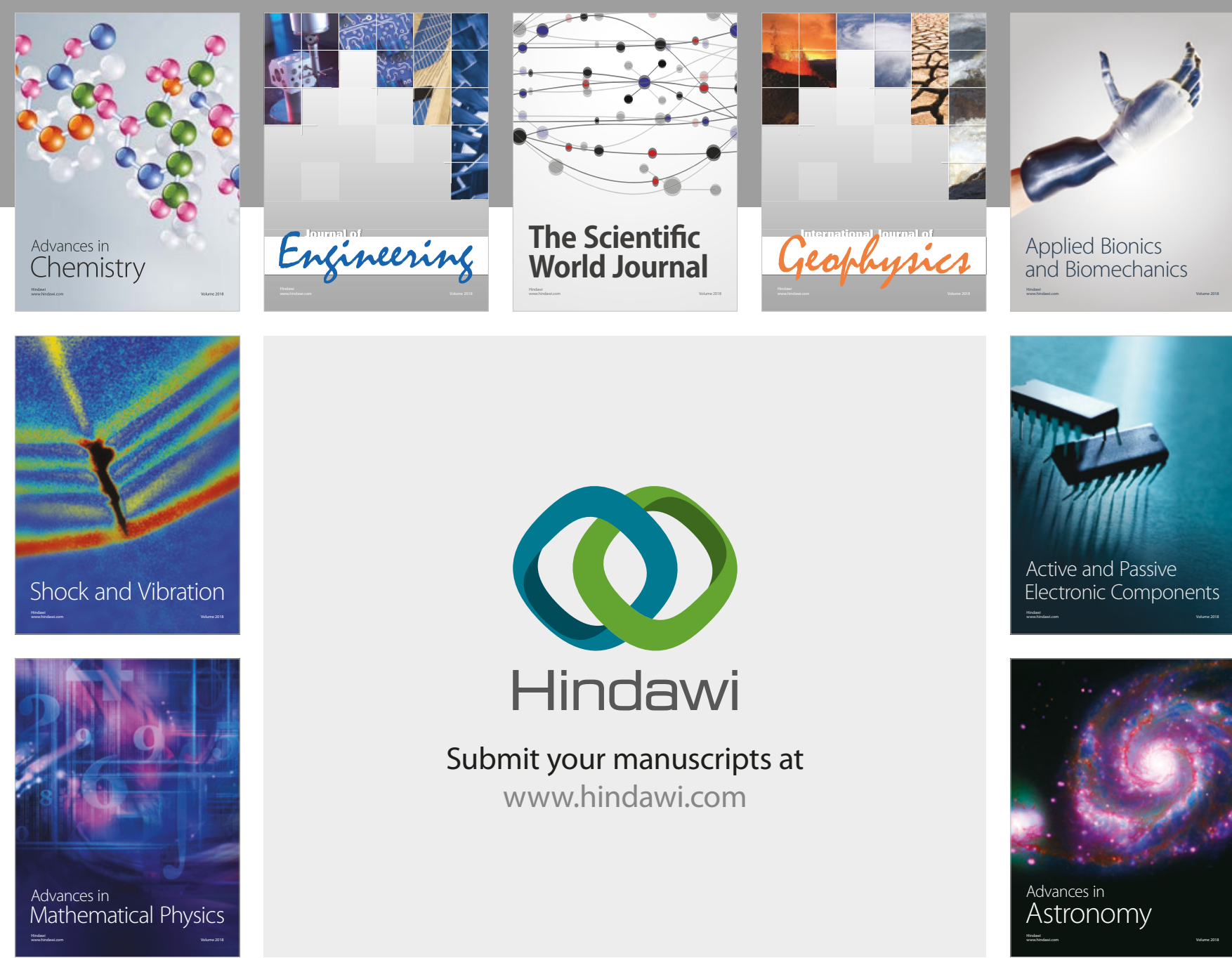

Submit your manuscripts at

www.hindawi.com

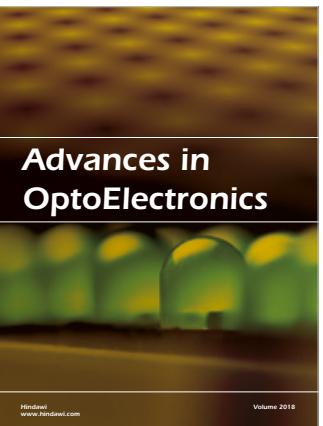

\section{Rotcting Machinery}
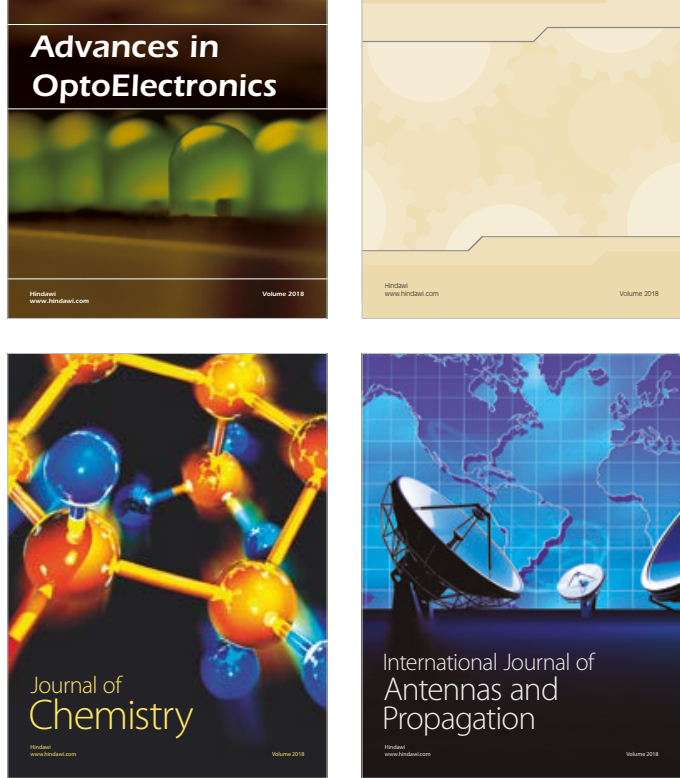

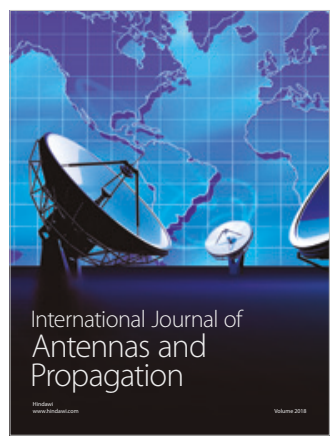

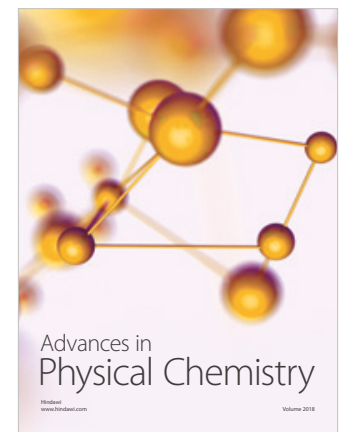

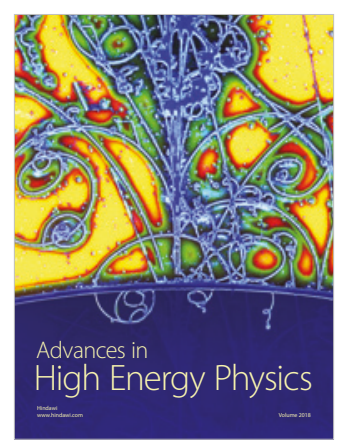

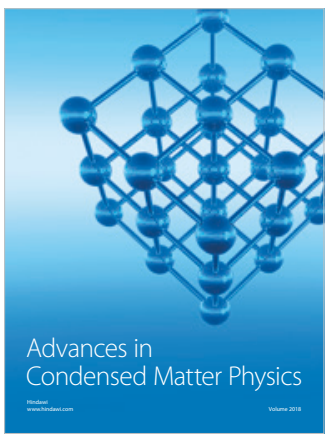

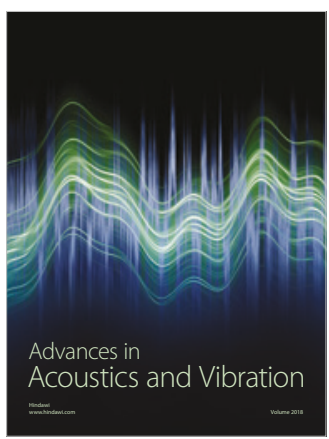

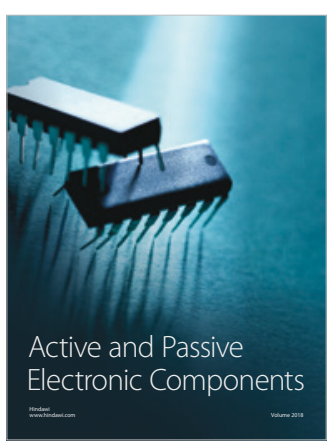
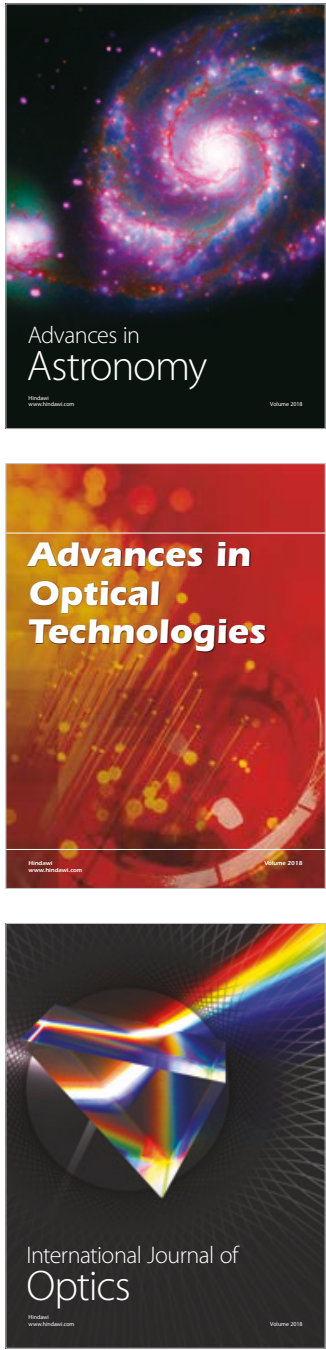